\title{
Unternehmerisches Finanzmanagement - Meilensteine der Entwicklung
}

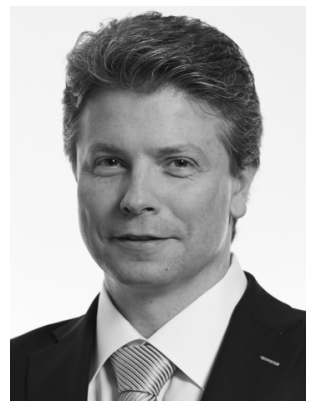

\section{Pascal Gantenbein}

Unternehmensbewertung, Finanzierung, Risikomanagement, Corporate Governance, Investor Relations, Entrepreneurial Finance

Corporate Valuation, Financing, Risk Management, Corporate Governance, Investor Relations, Entrepreneurial Finance

Im vorliegenden Beitrag werden die Kernthemen des heutigen unternehmerischen Finanzmanagements als zentrale Disziplin der Wirtschaftswissenschaften skizziert. Diese ist aus der früheren „Finanzierung" hervorgegangen. Die Basis bilden nach wie vor die beiden klassischen Bereiche Unternehmensbewertung und Finanzierung. Neuere Themenfelder stellen das Risikomanagement, die Corporate Governance sowie weitere Aspekte der finanziellen Führung dar. Als wesentliche Meilensteine in der Entwicklung der jeweiligen Konzepte ab dem 20. Jahrhundert werden die Orientierung an Kapitalmärkten, die Replikation von Anlagen im Hinblick auf die Bewertung sowie die Berücksichtigung von Aspekten asymmetrischer Information identifiziert. All diese Erkenntnisse haben in den genannten Themenfeldern eine Vielzahl neuer Entwicklungen sowohl in der Forschung wie in der unternehmerischen Praxis ausgelöst. Dazu zählt die Forschung zu Konglomeraten und zum Shareholder Value sowie zu Ankündigungseffekten und zur Kontrakttheorie. Zukunftsperspektiven lassen sich in den Bereichen Corporate Social Responsibility, Versicherungs-Risikomanagement und Pensionssysteme, Entrepreneurial Finance und Innovation sowie Finanz-Disintermediation und Crowd-Lösungen ausmachen, während sich Herausforderungen in der Praxis aus der Automatisierung von Bewertungs- und Finanzierungsdienstleistungen ergeben.

This article structures the core topics of what is known today as "Corporate Financial Management". This key discipline of business has evolved from corporate financing, but today comprises a much richer spectrum of topics. Corporate valuation on the one hand and corporate financing on the other still represent the backbone of the field. However, a number of further topic areas has grown over the years such as risk management, corporate governance, and investor relations. The analysis at hand focuses on the milestones and key drivers of this discipline's development since the early 20th century. Central milestones are identified as the inclusion of capital markets in research on investment and financing, the notion of replication for the purpose of valuation, and the consideration of different types of asymmetric information. All these findings and paradigms have contributed to a widening of further progress both in research and corporate practice. Examples include research on conglomerates and shareholder value as well as on announcement effects and contract theory. Future developments can be expected in the areas of corporate social responsibility, entrepreneurial finance, insurance risk and pension management as 
well as in financial disintermediation and crowd solutions. Further challenges may arise from the automation in valuation and financing services.

\section{Einleitung}

Das Gebiet des unternehmerischen Finanzmanagements in den Wirtschaftswissenschaften umfasst heute verschiedene finanzbezogene Themen im Tätigkeitsspektrum von Unternehmen. Die eigentliche Finanzierung im Sinne einer Zuführung von Eigen- und Fremdkapital für unternehmerische Prozesse ist dabei zwar nach wie vor ein zentraler Aspekt, doch hat sich im Verlaufe der vergangenen Jahrzehnte der Bogen an Themen substanziell erweitert. Vielmehr werden heute auch jene Aspekte und Fragen rund die Finanzierung einbezogen, die mit dieser in einem direkten oder indirekten Zusammenhang stehen. Der Begriff weist mithin gewisse Überschneidungen mit demjenigen der „Corporate Finance“ auf, der auch ausserhalb des angelsächsischen Sprachraums geläufig, jedoch stärker auf das Asset Pricing ausgerichtet ist.

Eine zentrale Voraussetzung praktisch aller finanzwirtschaftlichen Vorgänge in Unternehmen ist die Bewertung von Projekten, Vermögenswerten, Zahlungsströmen, Ansprüchen und Rechten, von Unternehmensteilen oder gar von ganzen Unternehmen. Eine Finanzierung unternehmerischer Vorhaben etwa ist nur möglich, wenn entweder eine ausreichende Werthaltigkeit von Aktiva, genügend hohe Freie Cashflows, ein spezifisches Wachstumspotenzial oder Forschungserkenntnisse in Aussicht stehen, die gegebenenfalls andernorts wertbringend eingesetzt werden können (wie zum Beispiel in der Biotechnologie).

Daher stellt die Bewertung heute das Fundament des Gebiets des unternehmerischen Finanzmanagements dar. Weitere Kernthemen umfassen neben der eigentlichen Finanzierung die Ausschüttungspolitik, das Zahlungs- und Risikomanagement sowie die Corporate Governance. Damit zusammen hängen sodann weitere Fragen rund um das Wertmanagement, die strategische Ausrichtung und die Portfoliogestaltung von Unternehmen, die Bewertung von Finanzinstrumenten und die Finanzanalyse sowie die Gestaltung von Investor Relations und den Einbezug weiterer Stakeholder im Rahmen der „Corporate Social Responsibility“. Ausgehend von den genannten Themenfeldern reicht das Spektrum somit deutlich weiter bis in die Organisation von Unternehmen betreffend Eigentümerstruktur und Verantwortlichkeiten.

Die Grenzziehung zu anderen funktionalen Bereichen der betrieblich orientierten Wirtschaftswissenschaften (wie etwa dem Controlling und dem Rechnungswesen) ist hierbei nicht immer scharf. Im Hinblick auf die deshalb bewusst relativ eng skizzierten Hauptgebiete werden nachstehend die zentralen Meilensteine des unternehmerischen Finanzmanagements nachgezeichnet und strukturiert. Der Fokus liegt hierbei auf den Entwicklungen seit Beginn des 20. Jahrhunderts und insbesondere auf den Erkenntnissen und Beiträgen seit den 1930er Jahren, die unser heutiges Verständnis in wesentlichem Masse geprägt haben. Für die zeitlich davor liegenden Entwicklungen der Finanzlehre seit dem Mittelalter sei hier auf Jonathan Barron Baskin \& Paul J. Miranti Jr. (2008) verwiesen. Die nachstehende Abbildung gibt eine Übersicht über die Kernthemen des vorliegenden Beitrags. 


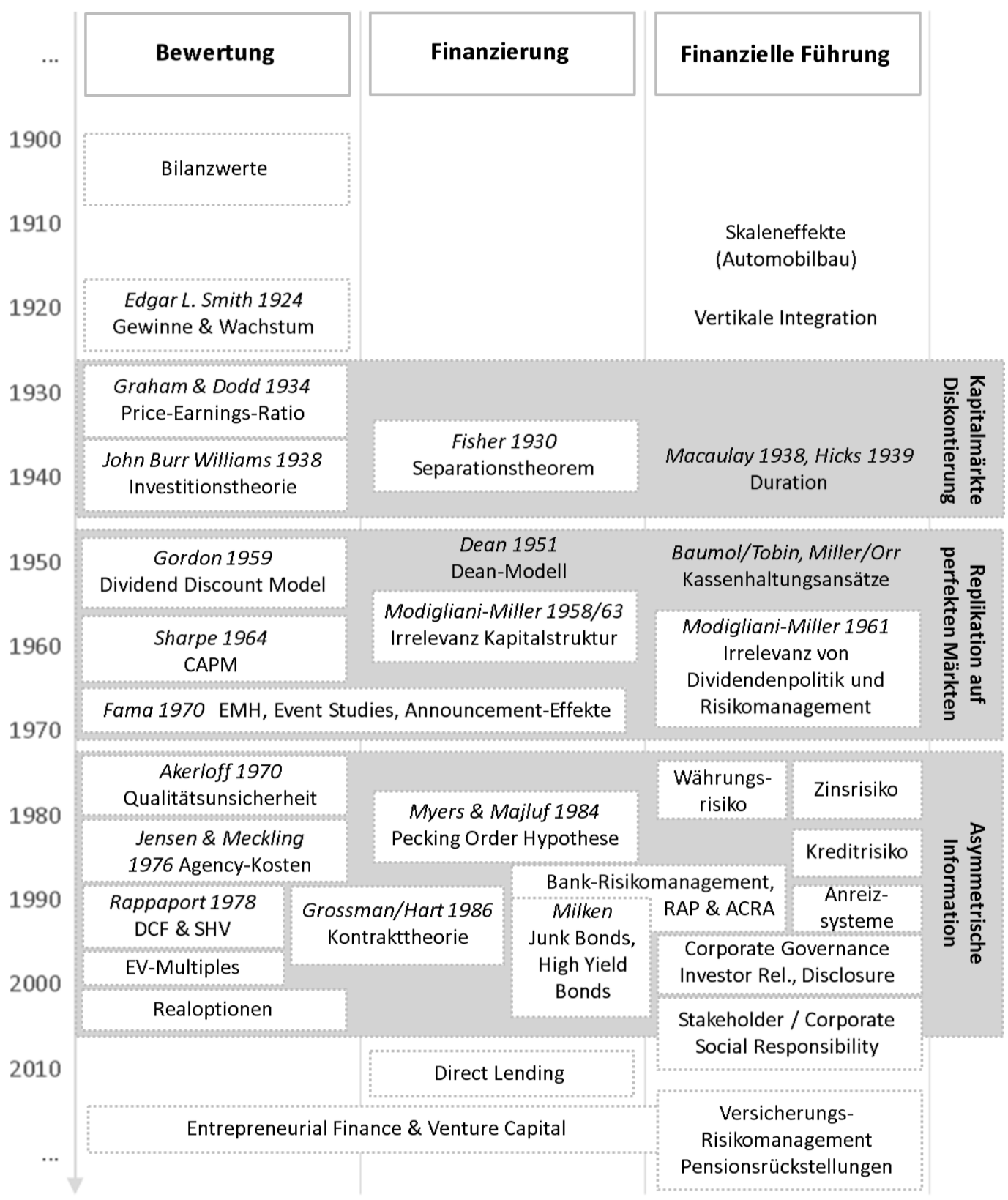

Abbildung 1: Meilensteine des unternehmerischen Finanzmanagements

\section{Meilensteine der Unternehmensbewertung}

Das Thema Unternehmensbewertung ist seit Ende des 19. Jahrhunderts durch mehrere Paradigmenwechsel gekennzeichnet gewesen (vgl. Spremann, 2004). War bis in die 1920er Jahre hinein die Orientierung an den Vermögenswerten (bilanzielle Sicht) verbreitet, hat sich in den Jahrzehnten danach eine immer stärkere Orientierung an den zukünftigen Zah- 
lungsströmen, die mit einer Investition verbunden sind, durchgesetzt. Diese unterscheiden sich je nach Bewertungsziel und -objekt: Sowohl bei Aktien (Dividenden, Gewinne pro Aktie, Freie Cashflows), Unternehmen (EBITDA, Freie Cashflows der Unternehmung), Krediten und Anleihen (Zinszahlungen bzw. Coupons, Amortisationszahlungen), Forschungsprojekten (Upfront Payments, Milestone Payments, Royalties) wie bei Immobilien (Mieteinnahmen), um nur einige zu nennen, leitet sich der Wert indessen immer aus der Zahlungsbereitschaft einer grösseren Menge an Käufern für einen in der Zukunft erwarteten Rückfluss ab. Zentral ist mithin die Orientierung an einem Fundamentalwert, also jenem Wert, der aus dem Investment heraus selbst generiert wird. Zusätzlich gelangen auch Vergleichswertverfahren zur Anwendung, die sich stärker an den in vergleichbaren Fällen bezahlten Preisen orientieren.

\subsection{Fundamentalwert und Finanzkennzahlen}

Ein früher Vertreter der Orientierung am Fundamentalwert war Edgar Lawrence Smith (1924). Smith hatte vorgeschlagen, für die Unternehmensbewertung auf die zukünftig erwarteten Unternehmensgewinne abzustellen und darüber hinaus deren Wachstum mit einzubeziehen. Grundlage hierfür war seine Arbeit mit einem Vergleich von Aktien- und Bondrenditen im Zeitraum von 1856-1923, in der er die im Vergleich zu den Bondrenditen höheren Aktienrenditen mitunter auf das Wachstum der Unternehmen zurückgeführt hat.

Nicht zuletzt vor dem Hintergrund der ab 1929 einsetzenden Weltwirtschaftskrise wurde dieses Paradigma nur wenige Jahre später durch einen weiteren Meilenstein in der Unternehmensbewertung abgelöst: 1934 veröffentlichten Benjamin Graham \& David Dodd die erste Ausgabe ihres Werks „Security Analysis“, einem heutigen Klassiker der Unternehmensbewertung. Wie Smith orientierten auch sie sich nicht an der Bilanz sondern an den Gewinnen. Im Unterschied zu Smith wird indessen kein Gewinnwachstum einbezogen sondern auf die Gewinne der letzten drei bis fünf Jahre abgestellt, mithin auf Grössen also, deren Erreichbarkeit die Unternehmen schon bewiesen haben. Im Falle einer unendlichen Gewinnreihe ergäbe sich der Aktienpreis somit aus $P=E / R$, mit $P=$ Aktienpreis heute, $E=$ Gewinne pro Aktie in der nächsten Periode (Earnings per Share) und $R=$ Diskontsatz bzw. Eigenkapitalkosten der Unternehmung.

Durch Umformung entsteht die Gleichung $P / E=1 / R$. Graham und Dodd haben damit nicht nur die heute in der Finanzanalyse verwendete Price-Earnings-Ratio (Kurs-GewinnVerhältnis, $K G V)$ als Vergleichskennzahl begründet sondern auch gezeigt, in welchen Grössenordnungen diese (aufgrund des durch den Kehrwert des Diskontsatzes gegebenen fundamentalen Ankers) sein soll: Unter der Annahme einer unendlichen, jedoch nicht wachsenden Reihe von Gewinnen ergeben sich je nach Aktienmarktrendite Werte für das KGV von 10 (bei einer erwarteten Aktienrendite von 10\%) bis 20 (bei einer erwarteten Aktienrendite von 5\%). Je nach Risiko (und damit Renditeerwartung) fallen die P/E-Ratios somit im Industrie- oder Unternehmensvergleich unterschiedlich aus. Diese Ratios werden heute in der Finanzanalyse auf breiter Basis verwendet.

Nur kurze Zeit darauf publizierte John Burr Williams sein berühmtes Werk „The Theory of Investment Value“ (1938). Er hat darin die Wichtigkeit von Finanzmärkten bei der Preisbildung betont und postuliert, dass Bewertungen auf Basis des Barwertes (Present Value) der zukünftigen Zahlungsströme durchgeführt werden sollen. Er gilt mithin als einer der Mitbegründer der Fundamentalanalyse. Auf diesen Ideen der Fundamentalanalyse so- 
wie der P/E-Ratio aufbauend publizierte Benjamin Graham (1949) „The Intelligent Investor“, in welchem er den Fokus auf das „Value Investing“ legte.

Dass Wachstum in den Bewertungsmodellen eine Rolle spielen soll, wurde zwar schon in der Nachkriegszeit mit dem einsetzenden Wirtschaftswachstum und neuen Produkten und Dienstleistungen deutlich, ist jedoch erst später durch Myron J. Gordon (1959) explizit wieder aufgegriffen worden. Im Zentrum des von ihm vorgeschlagenen Dividend Discount Model steht ein einfaches Modell, welches beschreibt, welchen Preis eine Aktie hätte, wenn dieser Preis auf Basis der ab nächstem Jahr erwarteten Dividende ermittelt würde. Auf Dividenden abzustellen macht insofern Sinn als diese den unmittelbaren Zahlungsfluss darstellen, den Aktionärinnen und Aktionäre aus ihrem Investment erhalten. Die Dividenden werden zum risikoadäquaten Eigenkapitalkostensatz diskontiert. Zudem werde unterstellt, dass die Dividenden von Jahr zu Jahr mit einer Rate $g$ wachsen können, dies bis in die Unendlichkeit. Die Modellgleichung lautet somit: $P=D /(R-g)$ mit $D=$ Dividendenerwartung im nächsten Jahr. Zu beachten ist hierbei, dass alle Grössen sich auf die Unendlichkeit der Reihe beziehen. Dadurch ist sichergestellt, dass $R>g$ gilt, dass also die risikoadäquate Aktienrendite die langfristige Dividendenwachstumsrate übersteigt.

Das Modell wird zwar heute kaum mehr in dieser Form verwendet, da Dividenden als wenig taugliche Grösse für Aktienbewertungen angesehen werden. Allerdings ist die Struktur des Modells heute in leicht modifizierter Form zu einem Standard in Unternehmensbewertungen geworden. Die Verbindung der aus dem Dividendenmodell folgenden Logik mit der von Graham und Dodd stammenden Überlegung, dass nicht Dividenden, sondern Gewinne (also auch deren nicht ausgeschüttete Teile) für die Aktionäre relevant sind, führt auf die Gleichung $P=E /\left(R-g_{E}\right)$ mit $g_{E}=$ Gewinnwachstum. Die Bewertung kann mithin vorteilhafterweise unabhängig von der Ausschüttungspolitik modelliert werden. Ein weiterer Vorteil dieses Modells besteht darin, aus den bekannten Kursen und Gewinnen kotierter Unternehmen auf die in den Aktienpreisen enthaltenen impliziten Gewinnerwartungen zu schliessen und daraus Informationen über die Investorenerwartungen abzuleiten.

\subsection{DCF und Shareholder Value}

Gewinne mögen zwar weniger stark verzerrt sein als Dividenden, doch stellen auch sie aufgrund von Abschreibungen und Debitorenverlusten keine gleichermassen repräsentative Basis dar wie Cashflows. In den 1970er und 1980er Jahren hat sich die weitere Entwicklung der Unternehmensbewertung daher stärker den Cashflows als Basisgrösse zugewandt und dabei insbesondere den „Freien Cashflows“. Hinzu kommt, dass die meisten realen Bewertungssituationen durch anfängliche Investitionen geprägt sind (Restrukturierungen, Unternehmensübernahmen im Rahmen von Nachfolgeregelungen) und daher in einer ersten Phase stark schwankende oder gar negative Cashflows generiert werden. Die Annahme einer bereits ab kommender Periode wirkenden unendlichen Zahlungsreihe kann somit bestenfalls eine grobe Approximation darstellen. Diese Aspekte wurden im Discounted Cashflow-Verfahren (DCF) berücksichtigt, welches mitunter durch Alfred Rappaport (1978, 1986) geprägt wurde.

Die Basis des DCF-Verfahrens stellen die Freien Cashflows dar, definiert als Cashflows abzüglich der erforderlichen Investitionen zur langfristigen Aufrechterhaltung der betrieblichen Produktion bzw. der wirtschaftlichen Leistungsfähigkeit. Diese Investitionen inkludieren auch Investitionen in das Anlage- und Umlaufvermögen im Zuge des Umsatz- bzw. Grössenwachstums (also Capital Expenditures und Erhöhungen des Netto-Umlaufvermö- 
gens). Der Horizont wird konzeptionell aufgeteilt in einen Planungshorizont (Planning Horizon) und einen Continuing Value. In ersterem werden schwankende Freie Cashflows pro Periode erfasst. Sobald mit einer gewissen Stabilität gerechnet werden kann, wird auch hier oftmals eine unendliche Reihe herangezogen, die nun die Form $C V=F C F F /\left(R_{W A C C}-\right.$ $\left.g_{F C F F}\right)$ hat, mit $C V=$ Continuing Value; $F C F F=$ Free Cashflow to the Firm (ab nachfolgender Periode), $R_{W A C C}=$ gewichteter Kapitalkostensatz, $g_{F C F F}=$ Wachstumsrate der FCFF. Auch diese Struktur ist mithin identisch mit jener des Dividendenmodells.

Darüber hinaus ist Rappaport mit dem Shareholder Value-Gedanken (SHV) verbunden, da auf Basis der Bewertungsmethode Werttreiber identifiziert werden können: Die Methode verdeutlicht, welche Veränderungen wie stark zur Wertgenerierung beitragen. Damit verbunden ist auch eine normative Aussage, nämlich dass das Management den Auftrag habe, den Wert ihres Unternehmens zu maximieren. Die damalige Entwicklung ist mitunter vor dem Hintergrund der Entstehung grosser, unübersichtlicher und ineffizienter Konglomerate in den 1960er und 1970er Jahren insbesondere im US-Markt zu sehen. Durch deren Übernahme und Aufspaltung konnten Corporate Raiders (darunter auch bekannte Namen wie z.B. Carl Icahn oder Ivan F. Boesky) nicht selten substanzielle Gewinne erzielen. Eine der bekanntesten Transaktionen (und bis heute der grösste Leveraged Buyout) war die Übernahme von RJR Nabisco durch Koblberg Kravis Roberts \& Co. (KKR) im Jahre 1988. Aus dieser Übernahmegefahr für schlecht aufgestellte Unternehmen und Gruppen folgte das Postulat, sich auf die Kernkompetenzen zu fokussieren statt zu diversifizieren. Mit der Resource Based View von Edith Penrose (1959) findet sich in gewisser Weise bereits ein Vorläufer dieses Gedankens.

Ebenfalls im Zusammenhang mit der Konglomeratsforschung wurde zunehmend das Problem asymmetrischer Information in den Bewertungsmodellen abgebildet. Während George A. Akerlof (1970) die Qualitätsunsicherheit thematisiert hatte, konnten Michael C. Jensen und William H. Meckling (1976) zeigen, dass aus einer Delegation von Entscheidungsrechten an das Management Agency-Kosten für die Eigentümer resultieren. Als Folge davon kann der Börsenwert eines Unternehmens tiefer sein als dessen intrinsischer Wert. In Bezug auf die Bewertungsverfahren sind auf dieser Basis weitere Varianten und Ergänzungen entstanden wie der Realoptionsansatz (vgl. Damodaran, 2000) und die Berücksichtigung von Intellektuellem Kapital (vgl. Damodaran, 2007) sowie Anpassungen der Modelle für Szenarioanalysen oder für die Bewertung von Start-ups. Häufig handelt es sich um Ergänzungen der DCF-Methode mit wahrscheinlichkeitsgewichteten Ergebnispfaden.

\subsection{Vergleichswertmethoden}

Die Ausführungen verdeutlichen, dass im Rahmen der beschriebenen Verfahren die Finanzmärkte und die dort explizit wie implizit verfügbaren Informationen für Unternehmensbewertungen so weit wie möglich als Orientierung einbezogen werden sollten. Finanzmärkte stellen eine zentrale Quelle der Information über Diskontraten, Wachstumserwartungen und Branchenspezifika bis hin zur Ableitung von Default-Wahrscheinlichkeiten und Kapitalkosten von Fremdkapitalinstrumenten dar sowie auch betreffend weiterer Aspekte (z.B. die Bewertung von Stimmrechten).

Es kann indes Situationen geben, in denen neben einer fundamentalen Bewertung auch interessiert, zu welchen Preisen Finanzaktiva gehandelt werden oder zu welchen Preisen Unternehmen von anderen Käufern übernommen worden sind. Zudem sind Berechnungen 
der Fundamentalwerte aufwändig, weshalb sich insbesondere Finanzinvestoren, zum Teil aber auch strategisch orientierte Investoren, vorzugsweise oder zusätzlich an Verhältniskennzahlen orientieren, welche den Unternehmenswert oder den Aktienpreis in Relation zu den Zahlungen oder auch zu beliebigen anderen Grössen setzen. Solche „Ratios“ werden als Multiples bezeichnet und dienen dazu, einen Unternehmenswert im Sinne eines Quervergleichs zu anderen Unternehmen mit potenziell ähnlichen Eigenschaften zu approximieren oder zu validieren bzw. um in der Finanzanalyse die Bewertungsunterschiede zu untersuchen und Kauf- oder Verkaufsgelegenheiten zu identifizieren.

Multiples stellen somit eine Form von Vergleichswertverfahren dar. Für Finanzinvestoren wurde mit der P/E-Ratio bereits ein solches Multiple erläutert. Typische Werte wurden zwischen 10 und 20 lokalisiert, bei hohen Wachstumserwartungen (wie um 1999/2000) oder bei sehr tiefem Renditeniveau (wie nach 2010) kann diese Ratio indessen vorübergehend auch deutlich darüber liegen. Eine weitere Kennzahl ist das Marktwert-BuchwertVerhältnis oder M/B-Ratio (vgl. hierzu Rhodes-Kropf, Robinson, Viswanathan, 2005). Diese bringt zum Ausdruck, ob der Marktwert des Eigenkapitals einer Unternehmung oberhalb oder unterhalb des Buchwerts liegt. Bei wissensintensiven, gesunden und wachsenden Unternehmen liegt dieser Wert zum Teil weit über 1, bei Unternehmen in Industrien mit viel Anlagekapital und wenig Wachstum zum Teil nahe oder gar unter 1.

Da sich in grösseren Transaktionen Finanzinvestoren (z.B. Private Equity-Häuser) ebenso wie strategische Investoren im Regelfall am Wert der Gesamtunternehmung orientieren, gelangen in diesen Fällen Multiples zur Anwendung, die sich auf den Enterprise Value (EV) beziehen. Dies macht auch deshalb Sinn, weil Käufer, die eine Kontrollmehrheit erwerben, oftmals mit Change of Control-Klauseln in der Finanzierung konfrontiert sind und die Zielunternehmung dann unabhängig von der bisherigen Kreditsituation neu finanzieren müssen.

Die häufigste Masszahl hierfür ist das EV/EBITDA Multiple (vgl. Liu, Nissim, Thomas, 2002; Koeplin, Sarin, Shapiro, 2000; Schreiner \& Spremann, 2007). Ein wichtiger Vorteil dieser Kennzahl besteht darin, dass sie unabhängig ist von der Abschreibungspolitik und daher vergleichsweise wenig verzerrt abbildet, welche Zahlungsbereitschaft die Käufer aufweisen in Bezug auf die operative Leistungsfähigkeit einer Unternehmung. Typische Werte liegen im Branchendurchschnitt etwa zwischen 6 und 9. Werte darüber wurden beispielsweise in den Jahren 2006-2008 sowie 2013-2015 verzeichnet und sind ähnlich wie die P/E-Ratio entweder Ausdruck hoher Wachstumserwartungen oder eines tiefen Renditeniveaus. Die grosse Herausforderung besteht jedoch in der Selektion der passenden Vergleichsgruppe von Unternehmen (vgl. Purnanandam \& Swaminathan, 2004; Paleari, Signori, Vismara, 2014).

\section{Meilensteine der Unternehmensfinanzierung}

Die in den Meilensteinen der Unternehmensbewertung aufgezeigten Paradigmen und Sichtweisen auf finanzwirtschaftliche Austauschbeziehungen sind auch in anderen Bereichen des unternehmerischen Finanzwesens manifest geworden. Dies gilt insbesondere für die Konzepte der Unternehmensfinanzierung, auf deren Basis später wiederum Ansätze zum Risikomanagement, zum Liquiditätsmanagement sowie zum Asset Pricing entwickelt wurden. 


\subsection{Annahme perfekter Kapitalmärkte}

Während Joel Dean (1951) im heute als „Dean-Modell“ bekannten Konzept noch eine Strukturierung der Finanzierungsmassnahmen nach ansteigenden Kapitalkosten propagiert hatte (unter der Annahme unterschiedlicher Finanzierungskonditionen), hat sich diese Sicht mit der zunehmenden Betonung von Kapitalmärkten grundlegend geändert. Wie bereits in der Fisher-Separation (nach Irving Fisher, 1930) erkannt, ist bei Existenz eines Kapitalmarktes, auf dem finanzielle Ressourcen angelegt und Kredite aufgenommen werden können, das Kapitalwertkriterium sowohl für die Produktionsentscheidung als auch für die Finanzierungsentscheidung zentral. Unter Annahme eines gut funktionierenden Kapitalmarktes ist deshalb nicht die zeitliche Staffelung von Ein- und Auszahlungen aus Investitionen und Finanzierungsmassnahmen wichtig für die Investitionsentscheidung, sondern ausschliesslich deren Kapitalwert. Denn auch langfristige Investitionen lassen sich mit kürzer laufenden Finanzierungsmassnahmen bewerkstelligen, wenn eine Anschlussfinanzierung über den Kapitalmarkt möglich ist.

Mit dem Wiedererstarken von Finanzmärkten in der Nachkriegszeit hat diese Sicht an Bedeutung gewonnen. Für verschiedene seit den 1950er Jahren entstandene Konzepte in der Finance erwies es sich zu Beginn als nützlich, von der Idee perfekter Kapitalmärkte auszugehen. Ziel war es hierbei nicht die Realität möglichst genau zu beschreiben, sondern durch die Abstraktion deutlich zu machen, wo gerade die wesentlichen Gründe in der realen Welt dafür liegen, dass die Prozesse eben anders verlaufen als in einer perfekten Welt. Mit dieser Annahme verbunden war die Erkenntnis einer zweiten wichtigen Eigenschaft von Kapitalmärkten, nämlich die der Replizierbarkeit sämtlicher Punkte einer durch Basisanlagen gegebenen positiven Beziehung zwischen Renditeerwartung und Risiko. Diese war nicht nur ein Kernelement von Andrew D. Roy (1952), Harry M. Markowitz (1952), James Tobin (1958) und William F. Sharpe (1964) in ihren Überlegungen zur Portfoliotheorie und zur Kapitalmarkttheorie. Der gleiche Gedanke lag auch der Argumentation von Franco Modigliani \& Merton Miller (1958 sowie 1963) zugrunde: Gemäss dem LeverageEffekt überträgt sich das der Geschäftstätigkeit einer Unternehmung inhärente Risiko im Ausmass der Verschuldung auf das Eigenkapital. Eine die Fremdkapitalkosten übersteigende Gesamtkapitalrendite vorausgesetzt führen Erhöhungen des Leverage zwar zu einer höheren Eigenkapitalrendite, zugleich aber auch zu entsprechend mehr Risiko.

Eine höhere Rendite ist somit kein Free Lunch, sondern wird immer mit einem höheren Risiko erkauft. Falls nun die Investoren gleichen Zugang zu den Kapitalmärkten haben wie die Unternehmen, können sie deren finanzpolitische Massnahmen durch das Eingehen von mehr oder weniger Leverage problemlos auch in ihren eigenen Portfolien selbst erzeugen bzw. rückgängig machen. Im einfachsten Fall gibt es nur einen Zinssatz, zu welchem angelegt und finanziert werden kann. Unter der Annahme, dass von Steuern abgesehen wird und es weder Konkurskosten noch asymmetrische Information gibt, generieren finanzpolitische Massnahmen von Unternehmen daher keinen Mehrwert für die Investoren. Diese können durch Anpassung ihrer eigenen Portfolien jede Position auf der Rendite-Risiko-Geraden replizieren. Mithin besteht wie bei der Wertschriftenlinie von Tobin ausgehend vom risikolosen Zinssatz ein positiver linearer Zusammenhang zwischen Rendite und Risiko.

Diese Erkenntnis lieferte die Basis für die Modigliani-Miller (MM) Irrelevanzthesen: Diese besagen, dass - immer unter den obigen Voraussetzungen - der Unternehmenswert erstens unabhängig ist von der Verschuldung und dass durch finanzpolitische Massnahmen 
seitens der Unternehmen kein Wert für die Anleger geschaffen wird. Die insgesamt an die Kapitalgeber zu leistenden Kapitalkosten hängen dabei zweitens vom Risikoprofil der Unternehmung ab, nicht aber von deren Kapitalstruktur. Die MM-Irrelevanzthesen wurden häufig als realitätsfremd missverstanden. Doch gerade dank dieser Abstraktion ist es den Autoren gelungen, die wesentlichen Treiber für die faktische Relevanz der Kapitalstruktur herauszuarbeiten: Nämlich Steuern, Konkurskosten, Informationsintransparenz. Darüber hinaus waren Modigliani und Miller zusammen mit Tobin und Sharpe auch Wegbereiter des Replikationsgedankens in der Portfoliotheorie und der Bewertung. Die zweite These hat später auf das Konzept der Risikoklassen geführt wie wir es heute z.B. aus dem Kreditrisikomanagement kennen.

Auf dieser Basis konnte gezeigt werden, dass unter realen Verhältnissen ein Trade-off zwischen den Vorteilen der steuerlichen Abzugsfähigkeit von Fremdkapitalzinsen (Tax Shield) und den mit höherer Verschuldung einher gehenden steigenden Konkurskosten resultiert (Trade-off-Ansatz). Darüber hinaus hatte das Konzept auch eine wichtige Strahlkraft für weitere Themen: Analog zu den Überlegungen zur Irrelevanz der Kapitalstruktur gibt es auch die Irrelevanz der Dividendenpolitik (vgl. Modigliani \& Miller, 1961) sowie die Irrelevanz des Risikomanagements. Erstere ist dann gegeben, wenn die Rendite einbehaltener Gewinne identisch ist mit den Wiederanlagemöglichkeiten der als Dividenden ausgeschütteten Gewinne am Kapitalmarkt, wenn es keine verzerrenden steuerlichen Effekte gibt und wenn mit der Dividendenpolitik keine Signaleffekte erzeugt werden. Analog resultiert die Irrelevanz des Risikomanagements unter der Voraussetzung gleichen Marktzugangs für Absicherungsmassnahmen sowohl für Unternehmen wie für Investoren, dem Fehlen steuerlicher Verzerrungen durch Risikomanagement-Massnahmen (etwa infolge von Gewinnglättung) sowie dem Fehlen von Konkurskosten (etwa bei Verzicht auf Hedging-Massnahmen seitens der Unternehmen). Auch diese Überlegungen dienen eben gerade dazu herauszuarbeiten, warum unter gewissen Rahmenbedingungen bestimmte Massnahmen sinnvoller sind als andere.

\subsection{Asymmetrische Information}

Wie bei den Bewertungsansätzen hervorgehoben waren die 1970er Jahre zunehmend durch die Thematisierung der Problematik asymmetrischer Information geprägt. Zum einen geschah dies konzeptionell: Es wurde postuliert, dass asymmetrische Information relevant sei für Investoren und mithin für Kapitalkosten sowie die Kommunikation von Unternehmen mit ihren Investoren. Wie bereits unter 2.2. kurz erwähnt haben Michael Jensen \& William Meckling (1976) in einem wegweisenden Beitrag den Zusammenhang zwischen Agency-Kosten und der Kapitalstruktur besprochen. Ein paar Jahre später folgte ein Aufsatz von Stewart C. Myers und Nicholas S. Majluf (1984), in welchem sie die empirisch bereits früher erkannte „Hackordnung “ (Pecking Order) der Finanzierung thematisierten.

Sowohl bei Jensen \& Meckling wie bei Myers \& Majluf stehen Finanzierungsmassnahmen im Zusammenhang mit asymmetrischer Information. Während erstere die PrinzipalAgenten-Problematik und die Agency-Kosten betonen und unter anderem zeigen, dass eine bestimmte Fremdkapitalquote einen disziplinierenden Effekt auf das Management hat, steht bei letzteren die Qualitätsunsicherheit im Zentrum. Diese impliziert, dass Investoren an den Finanzmärkten finanzpolitische Massnahmen von Unternehmen auch als Signale interpretieren und ihre eigenen Erwartungen entsprechend anpassen. So gründet die in der Pecking Order festgestellte Wahl von Eigenkapital als letzte Finanzierungsoption bei einer 
Kapitalbeschaffung nicht nur auf der Tatsache, dass dadurch Stimmrechte und Gewinne verwässert werden. Vielmehr wird die Emission von Eigenkapital als Signal dafür gesehen, dass die emittierende Unternehmung am Markt überbewertet sein dürfte. Die empirische Forschung hat hierzu verschiedentlich einen den EPS-Effekt (Verwässerung der Earnings per Share) übersteigenden negativen Kurseffekt als Reaktion auf Eigenkapitalerhöhungen bestätigt.

Zum anderen erfolgte die Berücksichtigung von Informationsasymmetrie durch methodische Erweiterungen: Ausgehend von der als Benchmark dienenden Sicht der effizienten Märkte, welche von Harry Roberts (1959) und Eugene Fama (1970) geprägt wurde und wonach alle Informationen in den Kursen enthalten sein sollen, liessen sich mittels EventStudien auch Kursreaktionen auf spezifische Unternehmenstransaktionen messen. Die ersten Event-Studien wurden Ende der 1960er Jahre durchgeführt (vgl. Fama et al., 1969, Ball \& Brown, 1968) und eröffneten die Möglichkeit, die Auswirkung von Änderungen der Finanzierung und anderer unternehmerischer Charakteristika auf die Bewertung an den Finanzmärkten zu untersuchen. In den Jahren darauf bis heute folgte eine grosse Zahl an Untersuchungen zum Einfluss verschiedener Events auf das Kursbild von Aktien. Zum Thema Qualitätsunsicherheit entstanden zahlreiche Arbeiten, welche das Signalling etwa von Aktienkapitalerhöhungen, Kreditaufnahmen, Rating-Veränderungen, M\&A-Ankündigungen oder CEO-Wechseln untersucht haben, um nur die wichtigsten zu nennen. Häufig werden nicht die tatsächlichen Veränderungen sondern die Ankündigungen und somit die Announcement-Effekte analysiert.

Trotz der starken Thematisierung asymmetrischer Information mit ihren Ausprägungen Agency-Kosten, Qualitätsunsicherheit und Hold-up im genannten Zeitabschnitt gab es freilich schon frühere Arbeiten hierzu. Erinnert sei hier an Ronald Coase (1937), der bereits die Kontrollkosten innerhalb von Unternehmen berücksichtigt hatte.

\subsection{Entwicklungen der Unternehmenspraxis}

Wichtige Entwicklungen, die später Gegenstand der Forschung geworden sind, wurden auch durch veränderte Bedürfnisse an den Finanzmärkten und das Aufkommen neuer Finanzinstrumente geprägt. Euromärkte wurden in den 1970er Jahren durch die Nachfrage nach Dollar-Anlagen ausserhalb des Einflussbereichs der USA angetrieben. Aus den ersten noch bescheidenen Eurobond-Platzierungen 1961 (Sacor) und 1963 (Autostrade) entwickelte sich ein neues Marktsegment mit Eurogeldanlagen, Eurokrediten und Eurobonds. Als letztere werden Anleihen bezeichnet, die in einer anderen Währung als in jener des Emissionslandes emittiert werden.

Ein weiterer Meilenstein erfolgte mit der Erschliessung des High Yield Bonds-Marktes in den 1980er Jahren durch Akteure wie Michael Milken. War der Markt für hochverzinsliche Anleihen bis dahin hauptsächlich durch Unternehmen geprägt, die im Verlaufe der Zeit an Bonität eingebüsst haben (Fallen Angels), wurden in den 1980er Jahren zunehmend Sub-Investment-Grade-Anleihen originär begeben zwecks Finanzierung von Restrukturierungen, Leveraged Buyouts und Start-ups. Es zeigte sich, dass diese ursprünglich als Junk Bonds bezeichneten Papiere gegenüber ihrem erwarteten Verlust einen Excess Return generierten und daher trotz hoher sowie stark konjunkturabhängiger Ausfallraten in grösseren Portfolien interessant sein konnten (vgl. Weinstein, 1987). Interessant ist die damit einhergehende Sicht auf Kreditrisiken: Während bis dahin der Fokus hauptsächlich auf der Vermeidung von Kreditrisiken lag, rückte mit dem bewussten Eingehen solcher Risiken de- 
ren adäquate Bewertung bzw. die Kalkulation von Kreditrisikoprämien in den Vordergrund. Es begann sich die Sicht durchzusetzen, dass alles seinen Preis hat, auch schlechte Kreditrisiken.

Nach einer ersten Welle solcher Finanzierungen in den 1980er Jahren, in welchen Junk Bonds für grössere Unternehmensübernahmen eingesetzt wurden, führte insbesondere die Aufhebung des Trennbankensystems in den USA und die damit verbundene Möglichkeit zur direkten Verbriefung von Mezzanine-Tranchen zu einer weiteren Beflügelung dieses Marktes, bis zur Finanzkrise von 2007-2009. Heutige Treiber dieses Marktes sind nicht nur Unternehmenstransaktionen sondern auch die Regulierung um Basel III, welche ein „Deleveraging“ der Banken erwirkt und damit zu einer Verlagerung von Krediten auf Kanäle ausserhalb des Bankensystems wie dem Direct Lending beiträgt. Dieses wird in den angelsächsischen Märkten schon seit Jahrzehnten praktiziert, im Gegensatz zu Ländern mit einem traditionell stärkeren Bankensystem wie in Europa. Schliesslich leistet auch die lockere Geldpolitik der Jahre nach 2009 ihren Beitrag dazu, indem sie institutionelle wie private Anleger in Investments vermeintlich höherer Renditen treibt, die indessen auch mit höheren Risiken verbunden sind.

\section{Meilensteine der finanziellen Unternehmensführung}

Investition und Finanzierung sind nur auf perfekten Finanzmärkten voneinander unabhängige unternehmerische Entscheidungen. Auf realen, durch asymmetrische Information und Konkurskosten geprägten Märkten indessen sind weitere Aspekte zu berücksichtigen. Diese beschränken sich, wie Modigliani und Miller sowie die spätere Forschung gezeigt haben, nicht nur auf Steuern, sondern haben mit unterschiedlichem Marktzugang für verschiedene Marktteilnehmer, mit asymmetrisch verteilter Information und Verhaltensrisiken sowie mit dem temporären Versiegen von Finanzmärkten in Krisensituationen zu tun. Vor diesem Hintergrund sind die Themenbereiche Risikomanagement sowie Corporate Governance und Kommunikation von grosser Relevanz. Besonders ausgeprägt sind Agency-Problematik, Qualitätsunsicherheit und Hold-up schliesslich bei Start-ups, mit deren finanziellen Fragestellungen sich die Entrepreneurial Finance befasst.

\subsection{Risikomanagement}

Auch langfristig gute Geschäftsmodelle und Investments müssen zwischenzeitliche Durststrecken überstehen können. Erforderlich ist deshalb ein entsprechendes unternehmerisches Risikomanagement. In finanzieller Hinsicht stehen das Management von Zahlungsströmen und von Wertänderungen auf Aktiva und Passiva, also von Liquiditäts-, Preisänderungs- und Gegenparteirisiken im Vordergrund.

Zum Risikomanagement sind insbesondere seit den 1970er Jahren zahlreiche Forschungsbeiträge entstanden. Ein erster Schub kam mit dem Aufbrechen des fixen Wechselkurssystems von Bretton Woods, welches ab 1971/72 die Wechselkursrisiken für international tätige Unternehmen und Anleger zu einem wesentlichen Risikofaktor hat werden lassen und zudem mit neuen Zinsänderungsrisiken verbunden war. Als Reaktion darauf kam es kurze Zeit später zur Einführung neuer derivativer Kontrakte für Wechselkursund Zinsabsicherungen. Ab 1973 wurden an der Chicago Mercantile Exchange (CME) sieben Währungsfutures gehandelt, ab 1975 an der Chicago Board of Trade (CBOT) Zinsfutures. Weitere Währungs- und Zinsderivate folgten. 
Ein inzwischen grosser Literaturstrang hat sich schon früh dem Management von Währungsrisiken und den Kapitalkosten von multinationalen Unternehmen (Multinational Corporations, MNCs) angenommen, so etwa Alan C. Shapiro (1978a, 1978b) (vgl. auch Shapiro \& Rutenberg, 1976). Aus Unternehmensperspektive zeigten Clifford W. Smith und René M. Stulz (1985), dass Hedging wertschöpfend ist, wenn dieses es den Unternehmen ermöglicht, den optimalen Investitionspfad zu verfolgen ohne dass sie aufgrund kurzfristiger Marktänderungen davon abweichen müssen. Darüber hinaus kann Hedging zur Gewinnglättung (mit entsprechend positiven steuerlichen Effekten bei progressivem Steuertarif) oder zur Reduktion der Konkurswahrscheinlichkeit beitragen (vgl. Dufey \& Giddy, 1981).

Neben der Identifikation und Bewertung von mit Währungsschwankungen verbundenen Risiken stellt sich sodann die Frage, welche Massnahmen ergriffen werden sollen und bis zu welchem Grad. Typische Hedgingstrategien im Bereich des Währungsrisikomanagements reichen von natürlichem Hedging (etwa über Kontrakte sowie Ein- und Auszahlungen in gleicher Währung) über die Wahl von Produktionsstandorten und Portfolioanpassungen bis zum Eingehen derivativer Positionen (vgl. Spremann, 1995).

Eine zweite wichtige Risikokategorie sind die Zinsänderungsrisiken. Diese umfassen das Zinsneufestsetzungsrisiko sowie damit verbundene Basisrisiken (infolge imperfekter Replikation mit Absicherungskontrakten), überdies durch das Verhalten der Marktakteure ausgelöste Einkommens- und Werteffekte (vgl. Finma, 1999). Konzeptionell hatten schon Frederick R. Macaulay (1938) und John R. Hicks (1939) die Messung von Zinsrisiken mittels der Duration vorgeschlagen. Doch wurde das Thema erst in den 1970er Jahren wieder stärker aufgegriffen (vgl. Weil, 1973, sowie Cox, Ingersoll, Ross, 1979).

Zur Messung und zum Management von Zinsänderungsrisiken sind insbesondere in der Bankenliteratur seit 1985 gerade auch im deutschsprachigen Raum zahlreiche Beiträge entstanden (vgl. Schierenbeck, 1985). In den letzten Jahren ebenfalls wichtiger geworden ist die Literatur zu Zinsrisiken im Versicherungswesen sowie zum Asset-Liability-Management in Pensionsfonds. Treiber dieser Entwicklung sind nicht nur die zunehmende Orientierung an Marktwerten, sondern gleichermassen auch aufsichtsrechtliche Regelungen (Solvency II, Swiss Solvency Test SST) sowie Accounting Standards. So zwingt je nach Anwendungsbereich das Fair Value-Prinzip die Versicherungsindustrie zur Erfassung der Positionen nach Marktwerten.

Vergleichsweise wenig Beachtung gefunden hat demgegenüber das Thema Pensionsrückstellungen auf Unternehmensbilanzen - dies obschon die ökonomischen Risiken analog sind $\mathrm{zu}$ jenen im institutionellen Versicherungs- und Pensionsmarkt und obschon es sich im Falle kotierter Unternehmen dabei nicht selten um zweistellige Milliardenbeträge handelt. Erkannt hat das Problem indessen die Praxis, wo Pensionsrückstellungen bei Unternehmenstransaktionen schon heute einen wichtigen Aspekt einer Due Diligence darstellen und nicht selten auch zu einem „Deal Breaker“ werden können.

Eine dritte, bislang vor allem im Bankenbereich bedeutende Risikokategorie stellen die Kreditrisiken dar. War noch bis in die 1970er Jahre das Paradigma der Risikovermeidung die wichtige Leitlinie, wurde diese zunehmend durch die Sicht einer Risikokompensation abgelöst: Kredite auch unterschiedlicher Bonität sollen eingegangen werden können, aber mit entsprechender Kompensation für die damit erwarteten Verluste (Expected Loss, EL). Damit werden eine Adverse Selektion sowie eine Quersubventionierung schlechter Risiken durch gute Risiken vermieden. Modelle der Diskriminanz von guten und schlechten Risi- 
ken (vgl. Altman, 1968) wurden im Zuge dessen erweitert um Scoring-Modelle, welche eine präzisere Risikoklassifikation ermöglichen. Diese wiederum ist Voraussetzung für das Risk Adjusted Pricing (RAP). Im RAP hat jeder Kreditnehmer für den mit seinem Engagement verbundenen erwarteten Verlust selbst aufzukommen in Form von höheren Kreditrisikoprämien.

Auf Ebene des Kreditportfolios fand dieser Gedanke in den frühen 1990er Jahren seine Fortsetzung im Actuarial Credit Risk Accounting (ACRA). Die kumulierten Expected Losses werden dabei budgetiert, und Abweichungen von dieser Grösse stellen Unexpected Losses (UEL) dar. Im Schnitt sollen diese gleich Null sein, können in guten Jahren aber negativ und in schlechten Jahren positiv ausfallen. Deshalb muss die Bank eine entsprechende Verlustreserve vorhalten, deren Kosten ebenfalls in die Kreditkonditionen einberechnet werden müssen. Getrieben wurde diese Entwicklung massgeblich auch durch die Bankenregulierung, welche in den Paketen Basel I, II und III eine Kategorisierung von Kreditrisiken und eine entsprechende Unterlegung mit Eigenmitteln verlangt. Neue Gestaltungsmöglichkeiten für die Kreditportfolien haben sich nach etwa 2000 durch Kreditderivate eröffnet. Allerdings hat sich die Entwicklung dieses Segments infolge der Finanzkrise von 2008 verlangsamt.

Darüber hinaus dürfte das Thema Kreditrisiken inskünftig auch in anderen Bereichen eine Rolle spielen. Durch das Niedrigzinsniveau nach 2009 angeheizt und durch Mindestverzinsungsvorschriften gezwungen haben in den letzten Jahren zunehmend institutionelle Investoren in Europa damit begonnen in Kreditsegmente mit höheren Renditen zu investieren, die aber naturgemäss auch mit höheren Risiken verbunden sind. Neben dem High Yield Markt fallen darunter Kreditengagements, welche mittels Direct Lending (vgl. Abschnitt 3.3) arrangiert werden. Das Angebot an solchen ausserhalb von Bankbilanzen laufenden Krediten wiederum wird geschaffen durch die Reduktion der Bankbilanzen im Rahmen von deren Anpassung an Basel III. Hierzu gibt es bislang kaum Forschung und Erfahrungswerte, wie im Falle einer Erhöhung der Kreditrisiken damit umgegangen wird.

Schliesslich hat insbesondere seit der Finanzkrise von 2008 auch das Liquiditätsmanagement neue Beachtung gefunden, nachdem deutlich wurde, dass Unternehmen mit genügend finanziellen Mitteln vergleichsweise wenig vom Einbruch der Liquidität an den Geldmärkten betroffen waren. Geschäftsmodelle mit starkem Exposure in kurzfristigen Finanzierungen wie etwa im Detailhandel oder in Debitorenpositionen wie etwa im Fahrzeugleasing haben dagegen Veränderungen erfahren. Frühe konzeptionelle Beiträge zum Liquiditätsmanagement finden sich mitunter in den Kassenhaltungsansätzen von William J. Baumol (1952) und James Tobin (1956) (Baumol-Modell) sowie von Merton H. Miller und Daniel Orr (1966) (Miller-Orr-Modell). In grösseren Unternehmen mit mehreren Divisionen und Geschäftseinheiten gelangen zudem oftmals verschiedene Formen des CashPoolings zur Anwendung. Neben dem Management der Liquidität wurde in der Literatur auch der Wertbeitrag thematisiert, den eine Reduktion des Cash Conversion Cycle leisten kann. Berühmtestes Beispiel hierzu ist wohl die Computerfirma Dell (vgl. Farris \& Hutchinson, 2002). In der Forschung ist der Themenbereich des Cash Managements allerdings nach wie vor von relativ begrenzter Relevanz.

\subsection{Corporate Governance, Investor Relations, Corporate Social Responsibility}

Ein zweiter, ebenfalls aus der Problematik der asymmetrischen Information resultierender Themenkreis hängt mit der Delegation von Entscheidungsrechten in Kapitalgesellschaften 
zusammen. Im Rahmen einer kapitalmarktorientierten Unternehmensführung sind sowohl Kontrolle wie Information über Unternehmen erforderlich, mithin eine adäquate Corporate Governance sowie Investor Relations.

Die Forschung zur Prinzipal-Agenten-Problematik, welche im Rahmen der Ausführungen zu Unternehmensbewertung und -finanzierung bereits besprochen wurde, ging den späteren Beiträgen zu Anreizsystemen (vgl. Spremann, 1987, 1990) und zur Kontrakttheorie (vgl. Grossman \& Hart, 1986) voraus. Insbesondere in grösseren Kapitalgesellschaften zeigen diese Aspekte ihre Relevanz. Kapitalgesellschaften (z.B. Aktiengesellschaften) weisen einerseits den Vorteil der Skalierbarkeit und des Poolings kleinerer Beträge zwecks Realisierung selbst sehr grosser Vorhaben auf. Dazu braucht es andererseits eine Delegation von Entscheidungsrechten an die Geschäftsführung. Mithin resultiert ein gewisser Trade-off zwischen Aktionärsdemokratie einerseits und der Sicherstellung von strategischer Konsistenz und operativer Handlungsfähigkeit der Unternehmung andererseits.

Mit den Ansätzen zur Bewältigung dieses Spannungsfelds befasst sich die Corporate Governance. Hierunter fallen sowohl interne wie externe Kontrollmechanismen. Zu ersteren zählen Instrumente, mittels derer die Eigentümerschaft Einfluss auf die Art der Geschäftsführung ausübt, etwa über den Verwaltungs- bzw. Aufsichtsrat, über die Ausübung von Mitwirkungsrechten an der Jahresversammlung und über die Festlegung von Anreizsystemen (z.B. Management-Vergütung) (vgl. Hermalin \& Weisbach, 2003). Die bereits erwähnte Übernahmegefahr für schlecht gemanagte Unternehmen („Market for Corporate Control“), die von der Verschuldung sowie den Accounting Standards ausgehende Disziplinierung des Managements, die Arbeitnehmervertretung im Verwaltungs- bzw. Aufsichtsrat sowie der Wettbewerb am Absatzmarkt zählen demgegenüber zu den externen Kontrollmechanismen. Das Thema hat insbesondere seit der Finanzkrise von 2008 grosse Aufmerksamkeit erfahren. Ein wesentlicher Teil der heutigen Literatur hierzu stammt aus dem Bereich Law and Economics.

Zur kapitalmarktorientierten Unternehmensführung gehört indessen auch die Kommunikation mit Stakeholdern. Im Rahmen der auf die Kapitalgeber bezogenen Forschung zu Investor Relations hat sich beispielsweise gezeigt, dass eine bessere Disclosure zu tieferen Risikoprämien und damit zu tieferen Kapitalkosten beiträgt oder dass eine geringere Dispersion von Analystenschätzungen (z.B. dank einer besseren Investor Guidance) mit einem stabileren Kursbild einher geht (vgl. Scherbina, 2001; Diether, Malloy, Scherbina, 2002; Ciccone 2003).

Darüber hinaus wird heute von Unternehmen auch Kommunikation erwartet, die sich an weitere Stakeholder wendet, dabei nicht nur an Mitarbeitende und Geschäftspartner sondern auch die ganze Öffentlichkeit. Der Einbezug von Stakeholdern wurde in der Corporate Finance-Literatur allerdings schon in den 1980er Jahren thematisiert, etwa durch Bradford Cornell \& Alan C. Shapiro (1987). Diese Haltung wird heute als Teil der Corporate Social Responsibility verstanden. Auch dieser Bereich ist ein derzeit wachsendes Forschungs- und Lehrfeld mit Bezügen zum Finanzmanagement.

\subsection{Entrepreneurial Finance}

Ein drittes, relativ neues Gebiet in dem Zusammenhang stellt jenes der Entrepreneurial Finance dar. Diese befasst sich mit finanziellen Aspekten innovativer KMUs und von Jungunternehmen. Wesentliche Charakteristika von Start-ups und Innovationsprojekten sind die hohe Unsicherheit betreffend den Geschäftsplan sowie die starke Abhängigkeit des Ge- 
lingens von einem funktionierenden Unternehmerteam. Agency-Problematik, Qualitätsunsicherheit und Hold-up treten hier mithin besonders ausgeprägt in Erscheinung. Hinzu kommen spezifische Herausforderungen in Bezug auf Finanzierung, Eigentumsverhältnisse und die Investitionsbewertung, in der klassische Methoden oftmals nicht weiterhelfen.

Die Agency-Problematik ergibt sich aus dem zwingend grossen diskretionären Spielraum des Managements (häufig das Gründerteam) und der Unmöglichkeit einer Attribution des späteren Ergebnisses auf die Rahmenbedingungen einerseits und den Einsatz des Teams andererseits. Zudem besteht Unsicherheit darüber, wie gut das Team in einer Krisensituation funktioniert und ob sich für die Kapitalgeber ein Sitz im Verwaltungsrat des Unternehmens auszahlt. Damit zusammen hängt die Qualitätsunsicherheit betreffend Produkt, Risikofaktoren und dem Verhalten von Mitbewerbern. Schliesslich sehen sich sowohl Kapitalgeber wie Gründer einer Form gegenseitigen Hold-ups ausgesetzt: Die Unternehmung ist bei Kapitalerhöhungsrunden vom Verhalten des Kapitalgebers bzw. der Kapitalgeber abhängig, insbesondere wenn in einer früheren Finanzierungsrunde schon viele Stimmrechtsaktien abgegeben worden sind oder wenn die Tranche zur weiteren Wachstumsfinanzierung einem anderen Investor verkauft werden soll (z.B. von einem Business Angel an einen Venture Capitalist, vgl. Kerr, Lerner, Schoar, 2014). Umgekehrt kann der Kapitalgeber seine eingebrachten finanziellen und zeitlichen Ressourcen nur dann nutzen, wenn die Gründer kooperieren.

Diese Aspekte werden durch spezifische Konstellationen von Kapitalgebern, eine starke Abhängigkeit von einem oder von wenigen Produkten, die zum Teil starke Forschungsorientierung von Start-ups gerade in der Hochtechnologie, die Notwendigkeit von Zahlungen für IP-Rechte (Intellectual Property Rights) sowie durch spezifische Bedingungen von Nachfolgefinanzierungen noch akzentuiert. Für die Bewertung gelangen etwa Entscheidungsbaumverfahren oder die Harvard Methode (vgl. Zider, 1998) zur Anwendung. Das Gebiet der Entrepreneurial Finance hat bis dato insbesondere in der Praxis und der Lehre seine Bedeutung erlangt. Einen Überblick über die frühe Literatur vermittelt David J. Denis (2004).

\section{Schlussfolgerungen und Zukunftsperspektiven}

Im Rahmen dieses Beitrags wurde der Versuch unternommen, die Meilensteine in der Entwicklung des heutigen Gebiets des unternehmerischen Finanzmanagements im Rahmen der Wirtschaftswissenschaften seit Beginn des 20. Jahrhunderts zu strukturieren. Der Fokus lag hierbei auf den Kernthemen Bewertung, Finanzierung, finanzielle Unternehmensführung. Im Rahmen letzterer wurden die Themen Risikomanagement, Corporate Governance und Kommunikation sowie Entrepreneurial Finance beleuchtet.

In einer Welt perfekter Kapitalmärkte lassen sich Zahlungsflüsse aus Finanzierungen und solche aus Investitionen zeitlich voneinander trennen und separat evaluieren, da auch Zwischenfinanzierungen oder -investitionen problemlos realisierbar sind. Entscheidend ist mithin lediglich der Kapitalwert der Investition bzw. der Finanzierung. Diese Erkenntnis kann zweifellos als ein Meilenstein des Finanzmanagements gesehen werden, da sie das heutige Verständnis von Bewertungen jeglicher Art geprägt hat. Projekte, Finanzinstrumente und Immobilien werden heute gleichermassen mit Hilfe von Barwerten (Present $\mathrm{Va}$ lue) evaluiert bzw. die Vorteilhaftigkeit eines Investments mittels der Nettobarwerte (Net Present Value, NPV). Ebenso basieren sämtliche Fundamentalbewertungen (z.B. Dividend Discount Model, Ertragsbewertung, DCF-Verfahren) wie sie vom Grundgedanken her seit 
den 1930er Jahren für Unternehmensbewertungen angewendet werden sowie implizit auch einige Vergleichswertverfahren mit Multiplikatoren (z.B. P/E-Ratio, EV/EBITDA Multiple) auf diesem Gedanken. Irving Fisher (1930), Benjamin Graham \& David Dodd (1934) sowie John Burr Williams (1938) haben mit dem Separationstheorem, der Ertragsbewertung von Aktien und der Investitionstheorie massgeblich dazu beigetragen. Voraussetzung ist ein Kapitalmarkt, der das Risikoprofil der Investition im Diskontfaktor spiegeln kann.

Aus dieser Sicht folgte ein zweiter Meilenstein Anfang der 1950er Jahre, nämlich die Erkenntnis, dass sich verschiedene Rendite-Risiko-Profile aus Basisinstrumenten an den Finanzmärkten mittels Replikation konstruieren lassen. Eine erste Folge hiervon ist die Arbitragefreiheit auf liquiden Märkten. Zweitens ist das Replikationsprinzip damit zu einem zentralen Bestandteil jeder Bewertung geworden. Auf dieser Logik basierten nicht nur die Kapitalmarktlinie von James Tobin (1958) und das Capital Asset Pricing Model von William Sharpe (1964) sondern auch die Irrelevanzthesen von Franco Modigliani \& Merton Miller (1958, 1963). Sie alle gingen von einem Zinssatz und einer mit einem bestimmten Risiko verbundenen Anlage aus. Bei Tobin und Sharpe war diese das Marktportfolio, bei Modigliani-Miller die vollständig eigenfinanzierte Unternehmung. Das Prinzip ist aber dasselbe: Sämtliche Positionen auf der sich zwischen Zinssatz und Anlage ergebenden Rendite-Risiko-Linie lassen sich durch Replikation erreichen. Drittens zeigt sich damit auch, dass bei einer Replizierbarkeit dieser Rendite-Risiko-Profile durch alle Akteure am Finanzmarkt finanzpolitische Massnahmen von Unternehmen keinen Wert schaffen, weil jeder Punkt auf der Linie auch durch die Investoren selbst erzeugt werden kann, in ihren eigenen Portfolien. Folglich ist auch der Wert von Unternehmen unter gewissen Annahmen unabhängig von der Kapitalstruktur.

Gerade mit dieser provokanten Abstraktion von der Realität haben Modigliani \& Miller deutlich gemacht, welches die effektiv relevanten Aspekte für die Kapitalstruktur sind: Steuern, Konkurskosten und asymmetrische Information. Damit waren sie auch Wegbereiter für die weitere Erforschung der asymmetrischen Information, welche als dritter Meilenstein gesehen werden kann. Die Berücksichtigung der Prinzipal-Agenten-Problematik und der daraus folgenden Agency-Kosten, die Qualitätsunsicherheit sowie die Gefahr unfairen Verhaltens (Hold-up) hat das Gebiet der Unternehmensfinanzierung in mannigfaltiger Weise geprägt. Asymmetrisch verteilte Informationen sind für die Kontrakttheorie und die Konzeption von Anreizsystemen genauso relevant wie in der Konglomeratsforschung, im Shareholder Value-Konzept, in der Corporate Governance und der Kommunikation gegenüber Investoren und anderen Stakeholdern. Die Erkenntnisse von Modigliani \& Miller waren schliesslich auch wegweisend für die Relevanz des Risikomanagements, welche sich ebenfalls mit Konkurskosten, asymmetrischer Information und Steuern begründen lässt. Hervorgehoben wurden hier die Währungsrisiken, Zinsrisiken, Kreditrisiken und die Liquiditätsrisiken.

Prägend für die Entwicklung des Gebiets waren mithin die Annahme eines Kapitalmarkts, das Prinzip der Replikation sowie die Berücksichtigung der Implikationen asymmetrisch verteilter Information und der daraus entstehenden Verhaltensrisiken. Erwähnt sei an dieser Stelle, dass dem Replikationsprinzip in der Unternehmensbewertung auch heute ein zentraler Stellenwert zukommt, da stets nach Referenzpunkten wie impliziten Wachstumsraten, Kapitalkosten und Verhältnis-Kennzahlen in vergleichbaren Industrien und Unternehmen gesucht wird. Selbiges gilt für die Immobilienbewertung, im Rahmen derer etwa bei hedonischen Bewertungsverfahren die implizit in Transaktionspreisen ent- 
haltenen Schattenpreise verschiedener Eigenschaften von Immobilien ermittelt werden. Diese Prinzipien finden mithin heute ihre Anwendung auch in Bereichen, wo die Märkte nur beschränkt gut funktionieren.

Zukunftsperspektiven lassen sich in verschiedenen Bereichen lokalisieren:

(1) Ein zwar nicht mehr ganz so neues Thema doch mit wachsender Bedeutung und mit Bezügen zum Finanzmanagement stellt die Corporate Social Responsibility dar. Die Forschung hierzu steht wie dargelegt erst am Anfang.

(2) Eine wichtige Entwicklung findet zudem im Themenbereich Entrepreneurial Finance statt. Dieser kommt insbesondere angesichts der Zwangsläufigkeit von Innovation in Start-ups und KMUs für das langfristige Wachstum und den Wohlstand von Volkswirtschaften eine zentrale Rolle zu.

(3) Forschungsbedarf wurde ferner im Versicherungs-Risikomanagement und zum Thema Pensionsrückstellungen festgestellt. Dieser Themenbereich dürfte auch durch die Fehlallokationen von Ressourcen seitens institutioneller Investoren im Zuge der Niedrigzinspolitik und der wachsenden Kollektivierung des Vorsorgesparens inskünftig noch wichtiger werden.

(4) Eine bemerkenswerte Entwicklung zeichnet sich auch in Bezug auf Lösungen ohne Finanzintermediation ab. Als Beispiel wurde der Trend zur bilateralen Kreditvergabe (Direct Lending) genannt. Ein möglicherweise interessantes, wenngleich zurzeit noch verschwindend kleines Gebiet stellt das Crowdfunding dar. Ähnliche Ansätze gibt es im Bereich Crowd Insurance.

(5) In Bezug auf Unternehmensbewertungen und -finanzierungen schliesslich wird die $A u$ tomatisierung von Bewertungsprozessen und Finanzierungslösungen in Verbindung mit einer Integration von Informationen aus dem Rechnungswesen diesen Teil der Finanzdienstleistungsbranche vor neue Herausforderungen stellen.

Es versteht sich von selbst, dass die in diesem Beitrag beleuchteten Themenbereiche nicht abschliessend sind, sondern sich aus der bewusst vorgenommenen Eingrenzung des Analysebereichs ergeben.

\section{Literatur}

Akerlof, George A. (1970): The Market for "Lemons": Quality Uncertainty and the Market Mechanism. The Quarterly Journal of Economics, Vol. 84, No. 3, August 1970, 488-500.

Altman, Edward I. (1968): Financial Ratios, Discriminant Analysis, and the Prediction of Corporate Bankruptcy. The Journal of Finance, Vol. 23, No. 4, September 1968, 589-609.

Ball, Ray \& Philip Brown (1968): An Empirical Evaluation of Accounting Income Numbers. Journal of Accounting Research, Vol. 6, 1968, 159-178.

Baskin, Jonathan Barron \& Paul J. Miranti Jr. (2008): A History of Corporate Finance. Cambridge University Press, 2008.

Baumol, William J. (1952): The Transactions Demand for Cash: An Inventory Theoretic Approach. The Quarterly Journal of Economics, 1952, 545-556.

Ciccone, Stephen J. (2003): Forecast Dispersion and Error versus Size, Book-to-Market Ratio and Momentum: A Comparison of Anomalies from 1992-2001. Journal of Asset Management, Vol. 3, No. 4, March 2003, 333-344. 
Coase, Ronald H. (1937): The Nature of the Firm. Economica, Vol. 4, No. 16, November 1937, 386-405.

Cornell, Bradford \& Alan C. Shapiro (1987): Corporate Stakeholders and Corporate Finance. Financial Management, Vol. 16, No. 1, Spring 1987, 5-14.

Cox, John C., Jonathan E. Ingersoll Jr., Stephen A. Ross (1979): Duration and the Measurement of Basis Risk. The Journal of Business, Vol. 52, No. 1, January 1979, 51-61.

Damodaran, Aswath (2000): The promise of real options. Journal of Applied Corporate Finance, Vol. 13, No. 2, 2000, 29-44.

Damodaran, Aswath (2007): Dealing with Intangibles: Valuing Brand Names, Flexibility and Patents. SSRN Paper, April 7, 2007.

Dean, Joel (1951): Capital Budgeting, Columbia University Press, 1951.

Denis, David J. (2004): Entrepreneurial finance: an overview of the issues and evidence. Journal of Corporate Finance, Vol. 10, No. 2, 2004, 301-326.

Diether, Karl B., Christopher J. Malloy, Anna Scherbina (2002): Differences of Opinion and the Cross Section of Stock Returns. The Journal of Finance, Vol. 57, No. 5, October 2002, 2113-2141.

Dufey, Gunter \& Ian H. Giddy (1981): Innovation in the International Financial Markets. Journal of International Business Studies, Vol. 12, No. 2, June 1981, 33-51.

Fama, Eugene, Lawrence Fisher, Michael Jensen, Richard Roll (1969): The Adjustment of Stock Prices to New Information. International Economic Review, Vol. 10, 1-21.

Fama, Eugene (1970): Efficient Capital Markets. A Review of Theory and Empirical Work. The Journal of Finance, Vol. 25, No. 2, 1970, 383-417.

Farris II, M. Theodore \& Paul D. Hutchinson (2002): Cash-to-cash: the new supply chain management metric. International Journal of Physical Distribution \& Logistics Management, Vol. 32, No. 4, 2002, 288-298.

Finma (1999): Rundschreiben der Eidg. Bankenkommission: Messung, Bewirtschaftung und Überwachung der Zinsrisiken (EBK-Rundschreiben 99/1 Zinsrisiko), 25.3.1999.

Fisher, Irving (1930): The Theory of Interest - As Determined by Impatience to Spend Income and Opportunity to Invest it. The Macmillan Company, 1930.

Gordon, Myron J. (1959): Dividends, Earnings and Stock Prices. Review of Economics and Statistics. The MIT Press, Vol. 41, No. 2, 1959, 99-105.

Graham, Benjamin \& David Dodd (1934): Security Analysis, Whittlesey House, 1934.

Graham, Benjamin (1949): The Intelligent Investor, Harper \& Brothers, 1949.

Grossman, Sanford J. \& Oliver D. Hart (1986): The Costs and Benefits of Ownership: A Theory of Vertical and Lateral Integration. Journal of Political Economy, Vol. 94, No. 4, August 1986, 691-719.

Hermalin, Benjamin E. \& Michael S. Weisbach (2003): Boards of directors as an endogenously determined institution: a survey on the economic literature. Economic Policy Review, Vol. 9, No. 1, 2003, 7-26.

Hicks, John R. (1939): Value and Capital: An Inquiry into Some Fundamental Principles of Economic Theory, 1939.

Jensen, Michael C. \& William H. Meckling (1976): Theory of the Firm: Managerial Behavior, Agency Costs and Ownership Structure. Journal of Financial Economics, Vol. 3, No. 4, 1976, 305-360. 
Kerr, William R., Josh Lerner, Antoinette Schoar (2014): The Consequences of Entrepreneurial Finance: Evidence from Angel Financings. The Review of Financial Studies, Vol. 27, No. 1, 2014, 20-55.

Koeplin, John, Atulya Sarin, Alan C. Shapiro (2000): The Private Company Discount. Journal of Applied Corporate Finance, Vol. 12, No. 4, Winter 2000, 94-101.

Liu, Jin, Doron Nissim, Jacob Thomas (2002): Equity Valuation Using Multiples. Journal of Accounting Research, Vol. 40, No. 1, March 2002, 135-172.

Macaulay, Frederick R. (1938): Some Theoretical Problems Suggested by the Movements of Interest Rates, Bond Yields, and Stock Prices in the United States since 1856. Columbia University Press, NBER, 1938.

Markowitz, Harry M. (1952): Portfolio Selection. Journal of Finance, Vol. 7, 1952, 77-91.

Miller, Merton H. \& Daniel Orr (1966): A Model of the Demand for Money by Firms. The Quarterly Journal of Economics, Vol. 80, No. 3, August 1966, 413-435.

Modigliani, Franco \& Merton H. Miller (1958): The Cost of Capital, Corporation Finance and the Theory of Investment. The American Economic Review, Vol. 48, No. 3, June 1958, 261-297.

Modigliani, Franco \& Merton H. Miller (1961): Dividend Policy, Growth and the Valuation of Shares. Journal of Business, Vol. 34, October 1961, 411-433.

Modigliani, Franco \& Merton H. Miller (1963): Corporate Income Taxes and the Cost of Capital: A Correction. The American Economic Review, Vol. 53, No. 3, June 1963, 433-443.

Myers, Stewart C. \& Nicholas S. Majluf (1984): Corporate financing and investment decisions when firms have information that investors do not have. Journal of Financial Economics, Vol. 13, No. 2, 1984, 187-221.

Paleari, Stefano, Andrea Signori, Silvio Vismara (2014): How Do Underwriters Select Peers When Valuing IPOs? Financial Management, Vol. 43, No. 4, Winter 2014, 731-755.

Penrose, Edith (1959): The theory of the firm. John Wiley \& Sons, 1959.

Purnanandam, Amiyatosh K. \& Bhaskaran Swaminathan (2004): Are IPOs Really Underpriced? The Review of Financial Studies, Vol. 17, No. 3, 2004, 811-848.

Rappaport, Alfred (1978): Executive Incentives vs. Corporate Growth. Harvard Business Review, Vol. 56, No. 4, 1978, 81-88.

Rappaport, Alfred (1986): Creating Shareholder Value: The new Standard for Business Performance. New York: Free Press, 1986.

Rhodes-Kropf, Matthew, David T. Robinson, S. Viswanathan (2005): Valuation waves and merger activity: The empirical evidence. Journal of Financial Economics, Vol. 77, No. 3, September 2005, 561-603.

Roberts, Harry (1959): Stock Market 'Patterns' and Financial Analysis: Methodological Suggestions. Journal of Finance, Vol. 44, 1959, 1-10.

Roy, Andrew D. (1952): Safety-First and the Holding of Assets. Econometrica, Vo1.20, 1952, 434-449.

Scherbina, Anna (2001): Stock Prices and Differences of Opinion: Empirical Evidence that Prices Reflect Optimism. SSRN Paper, April 2001.

Schierenbeck, Henner (1985): Ertragsorientiertes Bankmanagement: Betriebswirtschaftliche Grundlagen des Controlling in Kreditinstituten. Gabler, 1985.

Schreiner, Andreas \& Klaus Spremann (2007): Multiples and Their Valuation Accuracy in European Equity Markets. SSRN Paper, August 2007. 
Shapiro, Alan C. \& David P. Rutenberg (1976): Managing Exchange Risk in a Floating World. Financial Management, Vol. 5, No. 2, Summer 1976, 48-58.

Shapiro, Alan C. (1978a): Capital Budgeting for the Multinational Corporation. Financial Management, Vol. 7, No. 1, Spring 1978, 7-16.

Shapiro, Alan C. (1978b): Financial Structure and Cost of Capital in the Multinational Corporation. Journal of Financial and Quantitative Analysis, Vol. 13, No. 2, June 1978, 211-226.

Sharpe, William F. (1964): Capital Asset Prices: A Theory of Market Equilibrium under Conditions of Risk. The Journal of Finance, Vol. 19, No. 3, 1964, 425-442.

Smith, Clifford W. \& René M. Stulz (1985): The Determinants of Firm's Hedging Policies. Journal of Financial and Quantitative Analysis, Vol. 20, No. 4, December 1985, 391-405.

Smith, Edgar Lawrence (1924): Common Stocks As Long Term Investments, 1924.

Spremann, Klaus (1987): Agent and principal, in: Günter Bamberg \& Klaus Spremann (Hrsg.): Agency Theory, Information, and Incentives. Springer, 1987, 3-37.

Spremann, Klaus (1990): Asymmetrische Information. Zeitschrift für Betriebswirtschaft, Jg. 60, Nr. 5/6, 1990, 561-586.

Spremann, Klaus (1995): Das Management von Währungsrisiken, in: Henner Schierenbeck et al.: Handbuch Bankcontrolling, Springer, 1995, 837-862.

Spremann, Klaus (2004): Valuation: Grundlagen moderner Unternehmensbewertung. Oldenbourg, 2004.

Tobin, James (1956): The Interest-Elasticity of Transactions Demand for Cash. The Review of Economics and Statistics. Vol. 38, No. 3, August 1956, 241-247.

Tobin, James (1958): Liquidity Preference as Behavior Towards Risk. The Review of Economic Studies, Vol. 25, No. 2, 1958, 65-86.

Weil, Roman L. (1973): Macaulay's Duration: An Appreciation. The Journal of Business, Vol. 46, No. 4, October 1973, 589-592.

Weinstein, Mark I. (1987): A curmudgeon's view of junk bonds. The Journal of Portfolio Management, Vol. 13, No. 3, Spring 1987, 76-80.

Williams, John Burr (1938): The Theory of Investment Value. Harvard University Press 1938.

Zider, Bob (1998): How Venture Capital Works. Harvard Business Review, November-December 1998, 131-139.

Pascal Gantenbein, Prof. Dr., ist Professor für Finanzmanagement an der Wirtschaftswissenschaftlichen Fakultät der Universität Basel.

Anschrift: Universität Basel, Wirtschaftswissenschaftliche Fakultät, Peter Merian-Weg 6, CH-4002 Basel, E-Mail: pascal.gantenbein@unibas.ch 